\title{
Numerical modelling of radiating superorbital flows
}

\author{
$\begin{array}{lll}\text { R. J. Gollan } & \text { P. A. Jacobs* } & \text { S. Karl }\end{array} \quad$ S. C. Smith
}

(Received 8 August 2003; revised 4 February 2004)

\begin{abstract}
Experimental aerodynamic studies of the flows around new aerocapture spacecraft configurations are presently being done in the superorbital expansion tubes at The University of Queensland. Short duration flows at speeds of $10-13 \mathrm{~km} / \mathrm{s}$ are produced in the expansion tube facility and are then applied to the model spacecraft. Although high-temperature effects, such as molecular dissociation, have long been a part of the computational modelling of the expansion tube flows for speeds below $10 \mathrm{~km} / \mathrm{s}$, radiation may now be a significant
\end{abstract}

${ }^{*}$ Centre for Hypersonics, The University of Queensland, Brisbane 4072, Australia. mailto:rowan.gollan@uq. edu.au, mailto: peterj@mech.uq.edu.au

${ }^{\dagger}$ Institute for Aerodynamics and Flow Technology, German Aerospace Center, Göttingen, D-37073, GERmany

$\ddagger$ Centre for Computational Molecular Science, The University of Queensland, Brisbane 4072, Australia.

See http://anziamj.austms.org.au/V45/CTAC2003/Goll for this article, (c) Austral. Mathematical Soc. 2004. Published May 12, 2003, amended June 16, 2004. ISSN 1446-8735 
mechanism of energy transfer within the shock layer on the model. This paper will study the coupling of radiation energy transport for an optically thin gas to the flow dynamics in order to obtain accurate predictions of thermal loads on the spacecraft. The results show that the effect of radiation on the flowfields of subscale models for expansion tube experiments can be assessed by measurements of total heat transfer and radiative heat transfer.

\section{Contents}

1 Introduction

1.1 Expansion tube experiments . . . . . . . . . . . C251

2 Numerical Model

C252

3 Numerical Simulations

C253

3.1 Spherical bodies. . . . . . . . . . . . . . . C253

3.2 MUSES-C reentry vehicle . . . . . . . . . . . C C261

4 Concluding Remarks

C265

References

C266

\section{Introduction}

Future space exploration missions plan to use both aerocapture and aeroassist manouvres. During these flight trajectories it is known that radiative heating will become quite significant and the effect of radiation on the flowfield will be an important consideration. The presence of a radiating flowfield has two main effects on the prediction of heating on the forebody of a blunt body vehicle. Firstly, the radiation adds another mode of heat transfer to the 
vehicle surface. Along with convective heating, radiative heat transfer must also be considered to make an accurate heating prediction. The second effect of radiation in the shock layer is the so-called 'radiative cooling' effect. Some of the emitted energy escapes the shock layer and thus decreases the energy in the flowfield. The lowering of temperatures in the shock layer leads to an increase in the average density. This, in turn, produces a thinner shock layer. The thinner shock layer radiates less so ultimately radiative cooling reduces some of the effect of radiant heat transfer. It is apparent that the correct modelling of the fluid physics, the radiative transfer and their effects on each other becomes important for accurate heat load predictions.

Previous numerical studies of radiating flowfields concentrated on Earth entry. Sutton [15] provides an extensive review of the related work for air radiation. More recently, Matsuyama et al. [6] performed a numerical simulation of entry into the Jovian atmosphere which included radiation effects. Their calculation showed that radiative transfer was the major component of heat transfer for most of the entry trajectory. Olejniczak et al. [11] computed the heating environment for an aerocapture mission to Titan.

We investigate the modelling of radiating superorbital flows. We begin by describing the context for this work, namely the simulation of experiments on subscale aeroshell models performed in the superorbital expansion tubes at the University of Queensland [13, 8]. Next the numerical model of the radiating flow physics is outlined and the results of the numerical simulations are presented and discussed. To provide some confidence that the numerical model is reasonable, simulations of a sphere in low-density flow are compared with previous numerical experiments by Anderson [1]. Then simulations of a subscale model aeroshell are shown to have a much smaller fraction of radiative heat transfer than the full-scale flight vehicle. 


\section{$1.1 \quad$ Expansion tube experiments}

The superorbital expansion tubes developed at the University of Queensland [9] are used to simulate experimentally the flows encountered by hypersonic flight vehicles. The core diameter of the test flow is much smaller than many practical hypersonic vehicle dimensions and so subscale models of the flight vehicles are used. The experimental results are then scaled according to appropriate correlations to give realistic predictions for the actual flight vehicle.

A current interest for the experimenters is to test the validity of the scaling correlations related to radiative heat transfer to the surface of a blunt body entry vehicle. Experimenters are limited in the number and type of detailed measurements they can make of the flowfield under investigation. For example, in blunt body studies, it is typical to have measurements of pressure and heat transfer on the surface of the body. The use of heat transfer gauges allows for a measurement of total heat transfer to the vehicle surface at various locations. For flow visualisation, laser holography techniques are used at the Univeristy of Queenland [7]. From this flow visualisation method, it is possible to extract quantitative data about the mass density and electron density in the flow, and to obtain the measurement of shock stand-off distance.

Given that only a few features of the flow can be measured directly, it would be desirable for the experimenters to have some idea as to which flow features are significantly altered by the presence of radiation. The work here aims to use some simple numerical modelling of the radiating flows expected in the expansion tube experiments in order to provide initial estimates of various details of the flowfield. This will allow the experimenters to focus on those flow measurements which will give the best assessment of the level of radiation in the flow. 


\section{$2 \quad$ Numerical Model}

In this work, an axisymmetric compressible flow solver [5] is coupled with a model for the radiative heat transfer within the flow. This energy exchange appears as the divergence of the radiative flux, $\nabla \cdot \vec{q}_{r}$, and becomes a source term in the energy conservation equation of the axisymmetric Navier-Stokes equations. The full governing equations are presented and discussed in [4].

The computation of the radiative divergence, $\nabla \cdot \vec{q}_{r}$, would normally require a detailed solution of the radiative transfer equation. In this case, however, a simplification has been made such that the gas is considered optically thin (that is, it is transparent to the wavelengths of radiation that are being considered) and we assume that there is very little self-absorption of the radiation by the gas in the shock layer. This approximation is arguably appropriate for the flowfields about expansion tube models because the physical dimensions of the radiating shock layer are small compared to the scales for self-absorption.

Following Anderson [2], the radiative transfer equation is

$$
\frac{d I_{\nu}}{d s}=J_{\nu}-\kappa_{\nu} I_{\nu}
$$

Summing over all frequencies and all directions gives the divergence of the radiative flux,

$$
\nabla \cdot \vec{q}_{r}=\int_{0}^{\infty} \int_{4 \pi} J_{\nu} d \omega d \nu-\int_{0}^{\infty} \int_{4 \pi} \kappa_{\nu} I_{\nu} d \omega d \nu .
$$

For the optically thin assumption, the self-absorption term (the second term in equation (2) is omitted. The emission term can be integrated over all frequencies by using a mean absorption coefficient for the gas. The appropriate mean absorption coefficient for an optically thin gas is the Planck 
mean absorption coefficient

$$
\kappa_{P}=\frac{\int_{0}^{\infty} \kappa_{\nu} B_{\nu} d \nu}{\int_{0}^{\infty} B_{\nu} d \nu} .
$$

This determines the radiative divergence in terms of the Planck mean

$$
\nabla \cdot \vec{q}_{r}=4 \kappa_{P} \sigma T^{4}
$$

where $\sigma$ is the Stefan-Boltzmann constant. This is the numerical quantity that appears in the source vector and represents the 'radiative cooling' effect on the energy field. The Planck mean is a complex function of density and temperature of the radiating gas and involves detailed quantum mechanical modelling to compute an accurate value. For the purposes of this study, Olstad's [10] empirical relation is employed:

$$
\kappa_{P}=7.94\left(\frac{\rho}{\rho_{0}}\right)^{1.10}\left(\frac{T}{10^{4}}\right)^{6.95} .
$$

The numerical factor at the front has been changed so that $\kappa_{P}$ has units of $\mathrm{m}^{-1}$ instead of $\mathrm{ft}^{-1}$ and $\rho_{0}$ is sea-level density, taken to be $1.225 \mathrm{~kg} / \mathrm{m}^{3}$.

\section{Numerical Simulations}

\subsection{Spherical bodies}

To verify the numerical model, some calculations were performed of a test case presented by Anderson [1]. The conditions reported are for vehicles with nose radii of $0.3048 \mathrm{~m}(1 \mathrm{ft}), 0.6096 \mathrm{~m}(2 \mathrm{ft})$ and $1.2192 \mathrm{~m} \mathrm{(4ft)}$ at an altitude of $58 \mathrm{~km}$ and a flight speed of $15.24 \mathrm{~km} / \mathrm{s}$. This corresponds to a freestream pressure of $28.72 \mathrm{~Pa}$ and temperature of $252.5 \mathrm{~K}$. The gas model used in all simulations was air in thermochemical equilibrium. 


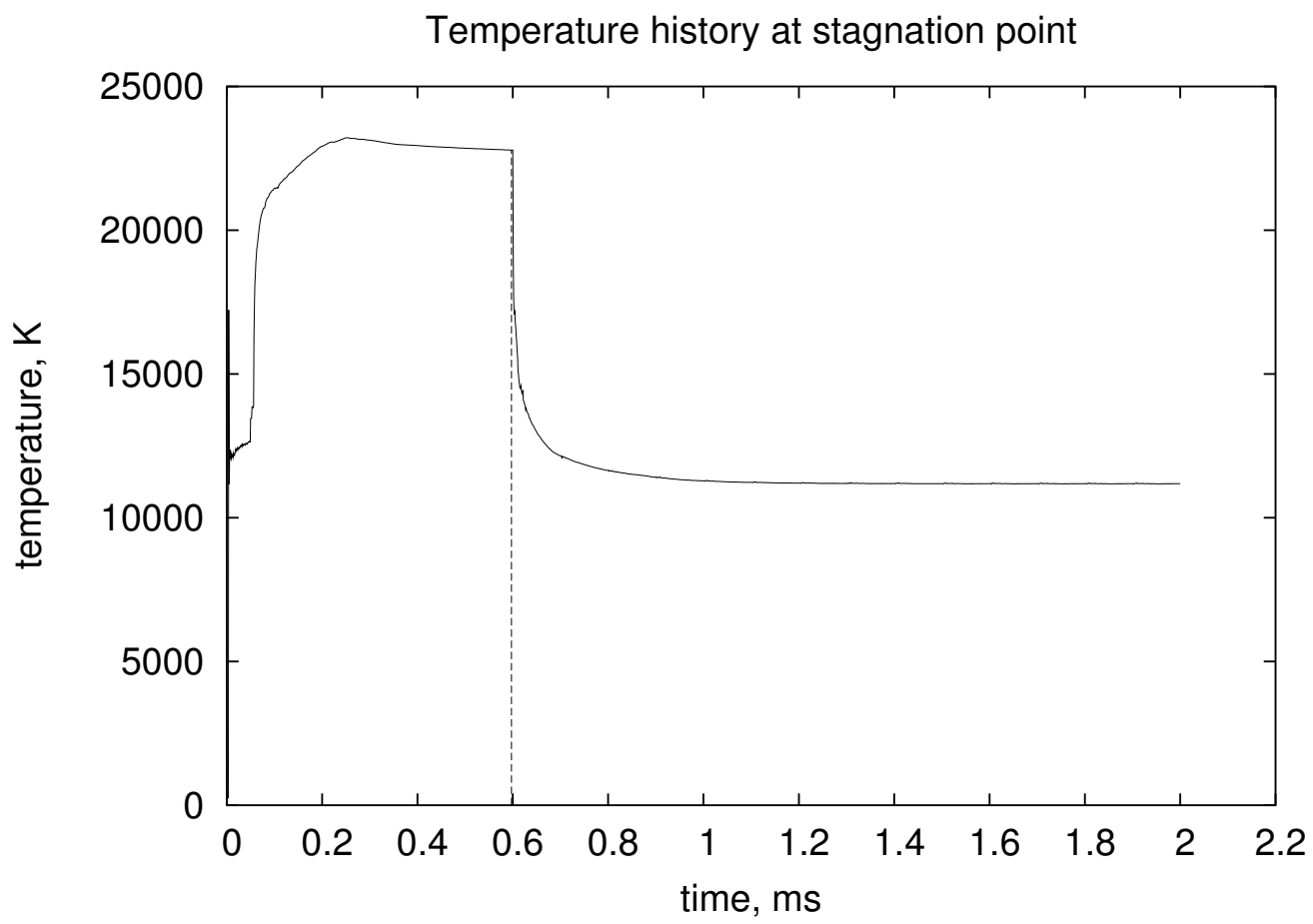

Figure 1: History of the temperature at the stagnation point for $r_{n}=$ $0.3048 \mathrm{~m}$ 


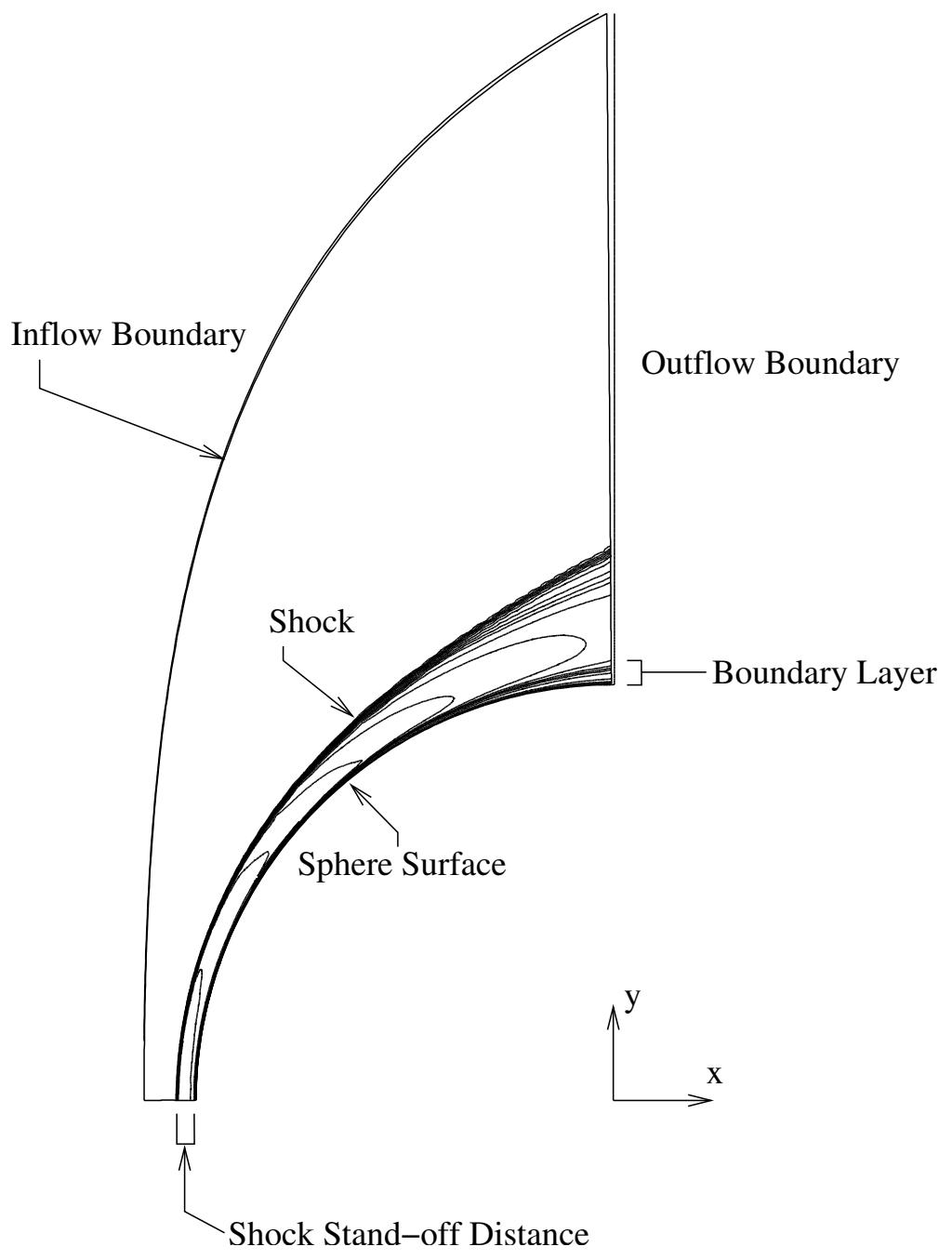

Figure 2: Contours of temperature for $r_{n}=0.3048 \mathrm{~m}$ 
For each of the present calculations, a converged flow solution is first computed without any accounting for the radiation effects within the flow. After the time taken for the flow to travel 30 times the distance of the body radius, the radiation effect is included in the calculation in a fully-coupled manner. This means that from this point in time, the energy loss due to radiation is computed in every finite-volume cell for every timestep. Figure 1 shows the history of the temperature at the stagnation point during the time-marching to steady-state for the nose radius of $0.3048 \mathrm{~m}$. The results for all nose radii are similar and the results for $r_{n}=0.3048 \mathrm{~m}$ are shown as a representative example. The vertical dashed line on Figure 1 shows the point in time $(t=0.6 \mathrm{~ms})$ at which the effect of radiation was included. The decrease of temperature at the stagnation point (and throughout the entire shock layer) once radiation is included is due to the 'radiative cooling' effect. Figure 2 shows the contours of temperature and gives some details of the flowfield for the same nose radius $r_{n}=0.3048 \mathrm{~m}$ when the effect of radiative cooling has been included and the simulation has marched to a final time of $t=2 \mathrm{~ms}$.

Figure 3 shows the results for shock stand-off distances for both the radiating and non-radiating cases. The results of Anderson [1] are also included for comparison. The present calculations do not agree exactly with Anderson's results but the discrepancy is small enough that we have confidence in the present model. Anderson's method involved an iterative shockfitting method based on finite-differences. By contrast, the present work uses time-marching shock-capturing techniques based on finite-volumes. In Figure 3 , see that the effect of radiative cooling is to reduce the thickness of the shock layer. Two extra points are computed in the present work: nose radii of $0.04 \mathrm{~m}$ and $0.02 \mathrm{~m}$. These points are computed because they represent the model sizes used in the University of Queensland expansion tubes [8].

The convective heating rates for varying nose radii are shown in Figure 4. The convective heating rate is calculated using the gradient of enthalpy at 


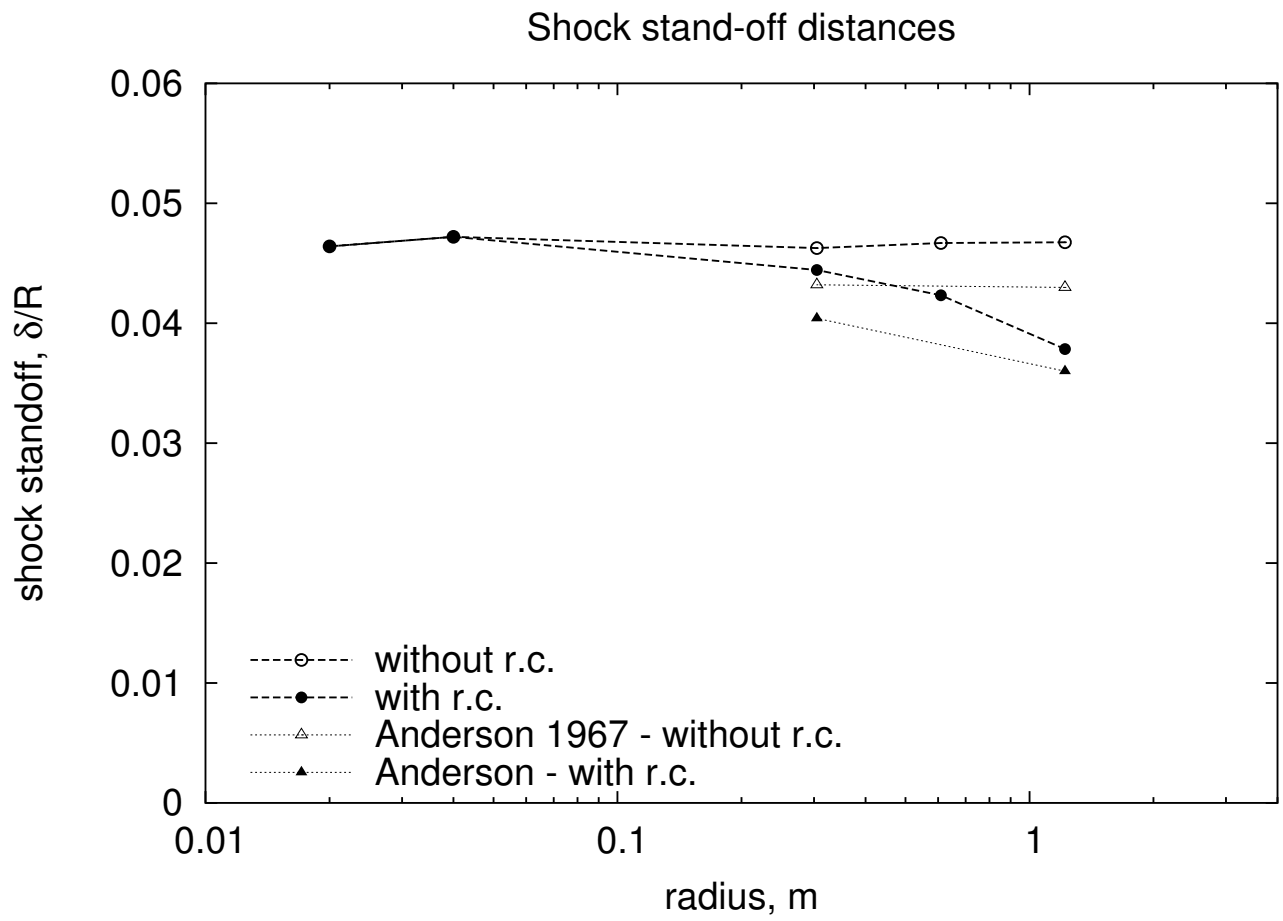

FiguRE 3: Shock stand-off distances non-dimensionalised with nose radius at $15.24 \mathrm{~km} / \mathrm{s}$ and an altitude of $58 \mathrm{~km}$. 'r.c.' denotes radiative cooling 
Convective heat transfer

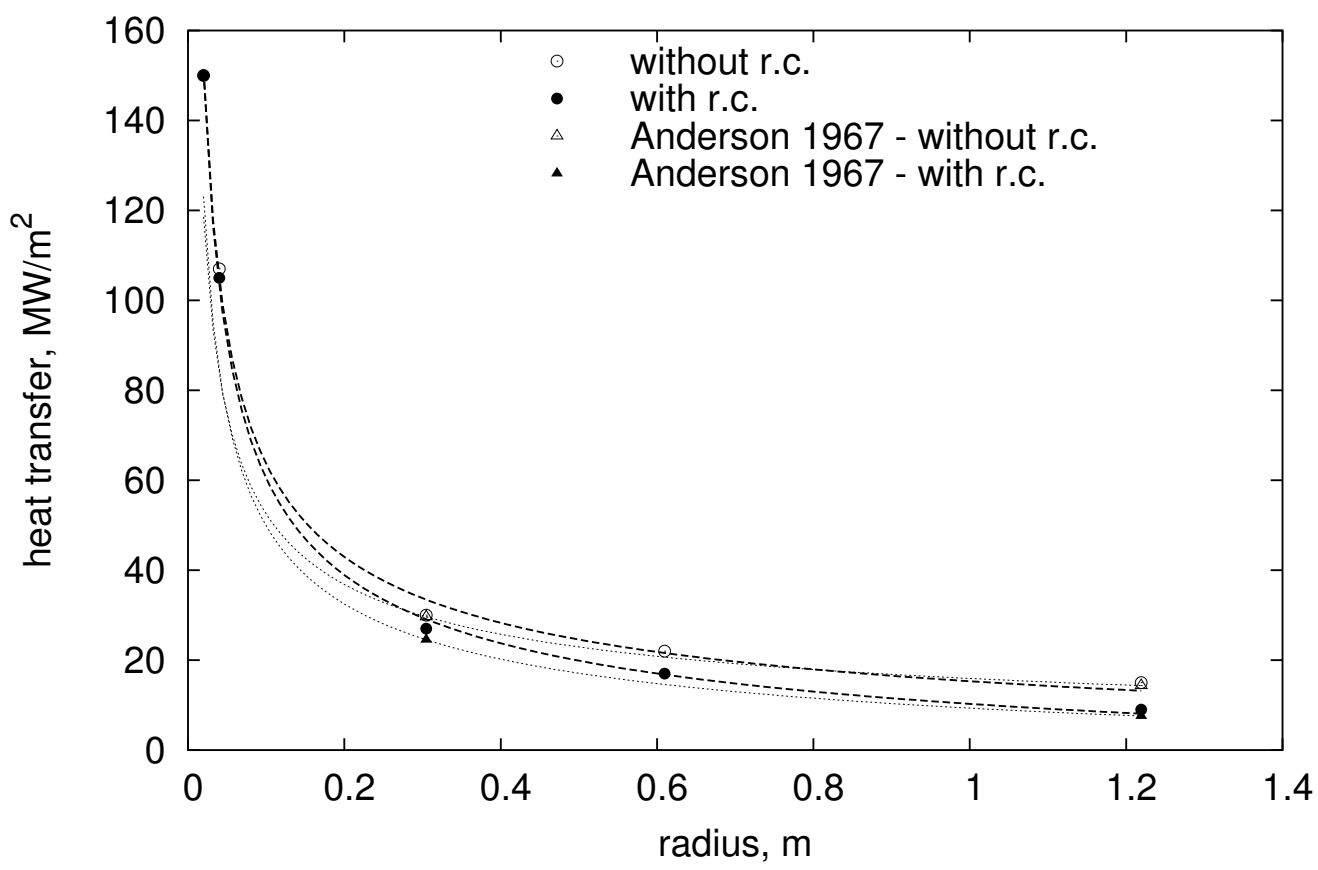

FIGURE 4: Convective heat transfer rate for varying nose radii at $15.24 \mathrm{~km} / \mathrm{s}$ and an altitude of $58 \mathrm{~km}$. 'r.c.' denotes radiative cooling 
the wall

$$
\dot{q}_{c}=\left.\frac{\mu}{\operatorname{Pr}} \frac{\partial h}{\partial y}\right|_{\text {wall }} .
$$

The correct computation of convective heat transfer requires a viscous solution with sufficient cell density to resolve the boundary layer and provide correct estimates of the temperature gradients approaching the wall. The data points for convective heat transfer are fitted with a curve that follows $r_{n}^{-1 / 2}$. Again the values do not exactly match those of Anderson [1] but they give confidence that the model is physically relevant. The 'radiative cooling' effect is again in evidence. It reduces the temperature in the shock layer and thus reduces the convective heating.

To compute the radiative component of heat transfer to the stagnation point, all the incident radiation onto the stagnation point surface must be included. This is based on the numerical integration of

$$
\dot{q}_{r}=\int_{V} \frac{J \cos \beta}{r^{2}} d V
$$

where $J$ is the radiant energy emitted per unit volume at every point in the flowfield, $r$ is the distance from the stagnation point to the emitting volume and $\beta$ is the angle between the surface normal of the stagnation point and the line out to the emitting volume. This equation is explained in more detail by Anderson [2, equation (18.14)]. The results are presented in Figure 5 and some comparisons given to Anderson's compuations [1] where available. Also an empirical correlation of Tauber and Sutton [16] is included over part of its range of validity. The present radiative heat fluxes are larger than Anderson's but this should be expected with a transparent gas model as there is no accounting for any attenuation of radiation between an emitting volume and the vehicle surface. The correlation of Tauber and Sutton does not agree well with either the present work nor Anderson's results - the problem of scaling radiative heat transfer is far from a settled question.

The interesting result in terms of heat transfer, shown in both Figures 4 and 5 is that, at the model sizes of interest for expansion tube experiments, 
Radiative heat transfer

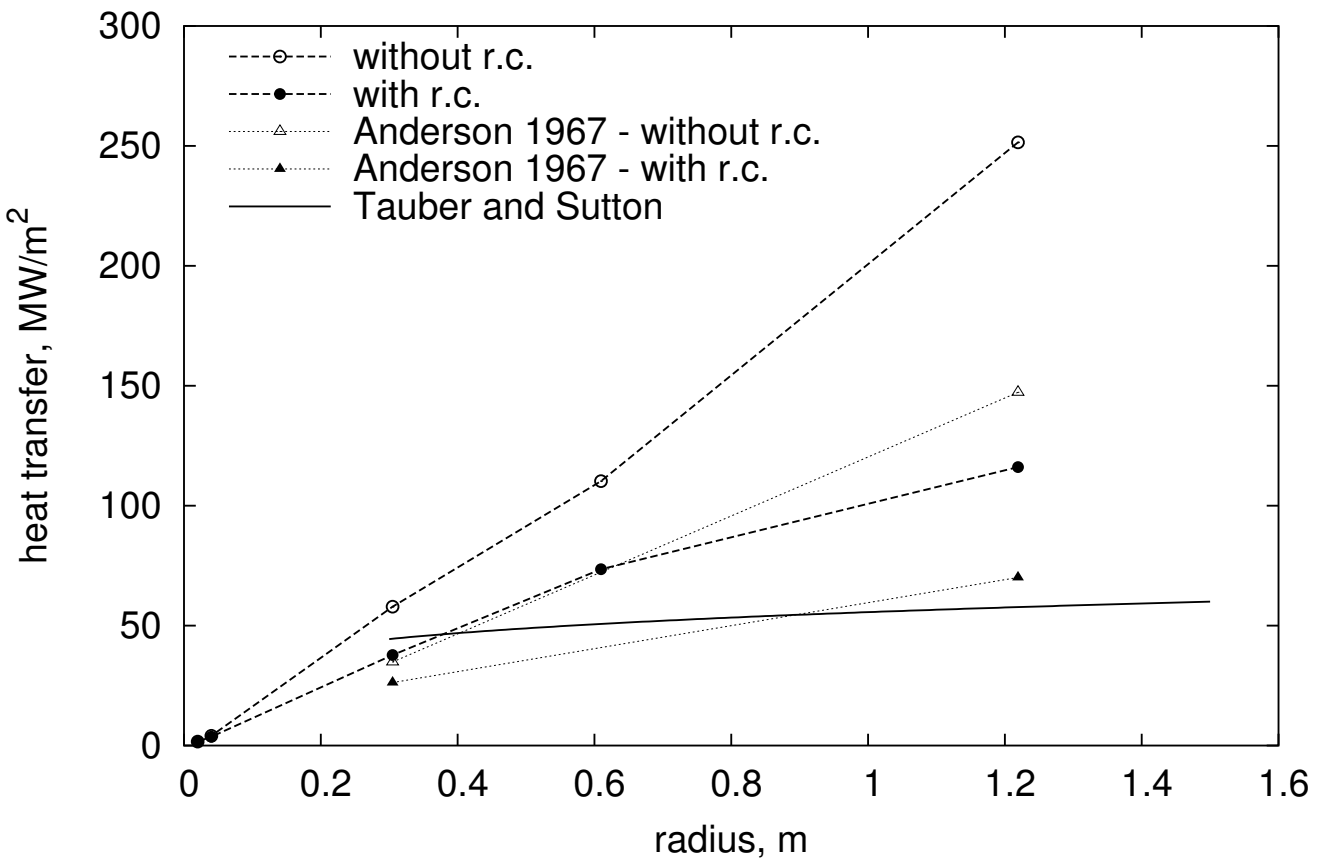

FIGURE 5: Radiative heat transfer rate for varying nose radii at $15.24 \mathrm{~km} / \mathrm{s}$ and an altitude of $58 \mathrm{~km}$. r.c. $=$ radiative cooling 
the radiative cooling effect has little effect on the measurable heat transfer rates. As an example, the stagnation point radiative heating for a nose radius of $0.02 \mathrm{~m}$ is $1.638 \mathrm{MW} / \mathrm{m}^{2}$ without accounting for radiative cooling in the flow and decreases to $1.628 \mathrm{MW} / \mathrm{m}^{2}$ with the radiation effect included. However, note that the radiative contribution to heat transfer is only $1 \%$ of the total and so do not place undue importance on this result because the free-stream density in these simulations is an order of magnitude smaller than that which is currently obtainable in the expansion tubes.

\subsection{MUSES-C reentry vehicle}

The MUSES-C reentry capsule is part of an asteroid sample return mission [12] and will reenter the Earth's atmosphere at approximately $12 \mathrm{~km} / \mathrm{s}$. The peak heat loading to the vehicle is predicted to be at an altitude of $65 \mathrm{~km}$ when its velocity is $11.6 \mathrm{~km} / \mathrm{s}$ [12]. The MUSES-C capsule design was investigated in the X2 superorbital expansion tube at the University of Queensland [8]. The experiment used test flow speeds of approximately $10.5 \mathrm{~km} / \mathrm{s}$. The simulation presented here uses the same model dimensions as the expansion tube experiment but alters the freestream conditions such that they better match the peak heating conditions expected in the actual flight. The results are preliminary as there are known deficiencies in the modelling at present.

At the reported altitude of $65 \mathrm{~km}$, the atmospheric density is $1.645 \times$ $10^{-4} \mathrm{~kg} / \mathrm{m}^{3}$ and the temperature is $233.25 \mathrm{~K}$. The predicted convective heat transfer is $7.5 \mathrm{MW} / \mathrm{m}^{2}$ and radiative heat transfer is $0.94 \mathrm{MW} / \mathrm{m}^{2}$ [3]. When simulating the freestream conditions of a flight vehicle in the expansion tubes, a scaling argument needs to be used to account for the differing vehicle dimensions. The well-known binary or ' $\rho L$ ' scaling argument [14, e.g.] is often used as it preserves the chemical kinetic scales in the flowfield so long as the velocity of the experiment matches the velocity of the vehicle. Based on this 
argument, the following freestream conditions are used for this simulation,

$$
\begin{aligned}
& \rho_{\infty}=1.645 \times 10^{-3} \mathrm{~kg} / \mathrm{m}^{3}, \quad T_{\infty}=233.25 \mathrm{~K}, \\
& p_{\infty}=110.12 \mathrm{~Pa}, \quad u_{\infty}=11.6 \mathrm{~km} / \mathrm{s} .
\end{aligned}
$$

The geometry for the MUSES-C entry capsule is a sphere-cone shape having a nose radius of $200 \mathrm{~mm}$, a cone angle of 45 degrees and a base diameter of $400 \mathrm{~mm}$. The simulated model is a 1:10 scale version of the flight vehicle (with a nose radius of $20 \mathrm{~mm}$ ). The wall temperature is set at $300 \mathrm{~K}$.

The converged radiating flowfield for the MUSES-C simulation is calculated in a similar fashion to the spherical body simulations in Section 3.1. Initially a converged flowfield is computed without any accounting for the radiation effects. Following this, the optically thin model for a radiating gas is coupled with the gas dynamics and the solution time-marched to a new converged flowfield.

The results of this simulation predict a convective heat transfer rate of $113 \mathrm{MW} / \mathrm{m}^{2}$ and a radiative heat transfer rate of approximately $1.2 \mathrm{MW} / \mathrm{m}^{2}$ to the stagnation point for both the case with radiative cooling and the case without. Using a scaling argument made by Zoby [17]

$$
\dot{q}_{c} \propto \sqrt{\frac{\rho}{r}} V^{3},
$$

for the convective heat flux and a form of the scaling relation for radiative heat flux presented by Tauber and Sutton [16]

$$
\dot{q}_{r}=C r^{a} \rho^{b} f(V),
$$

we compare to some similar experiments performed in expansion tubes by Palmer [13]. We change the value of the constant, $C$, used in the Tauber and Sutton [16] relation as the original published work is not valid below $r=0.3 \mathrm{~m}$. At condition 1 reported by Palmer [13], a total heat flux of $325 \pm 15 \mathrm{MW} / \mathrm{m}^{2}$ was measured at the stagnation point. The computed 
value here for convective heat transfer of $113 \mathrm{MW} / \mathrm{m}^{2}$ scaled to Palmer's condition 1 gives $\dot{q}_{c} \approx 307 \mathrm{MW} / \mathrm{m}^{2}$. The computed value for radiative heat transfer scales to $\dot{q}_{r} \approx 5.2 \mathrm{MW} / \mathrm{m}^{2}$ at condition 1 . This gives a scaled value of total heat transfer $\dot{q}_{\text {tot }} \approx 312 \mathrm{MW} / \mathrm{m}^{2}$ and lies within the uncertainty of the experimental measurement. It is difficult in this case to scale the actual flight vehicle to the subscale model (or vice versa) because the wall temperatures are different. The subscale simulation uses a wall temperature of $300 \mathrm{~K}$ which is representative of experimental conditions possible in an expansion tube. The simulations by Doihara and Nishida [3] have the wall temperature set at $2500 \mathrm{~K}$ to model the full scale vehicle. A simulation of the full scale vehicle using the present model and setting the wall temperature at $2500 \mathrm{~K}$ gives $9.3 \mathrm{MW} / \mathrm{m}^{2}$ for the convective heat transfer rate to the stagnation point. Although this value is $24 \%$ higher than that predicted by Doihara and Nishida [3] there are a number of differences in the thermochemical modelling which may account for this discrepancy. Specifically, the calculations here use a one- $T$ model rather than a five- $T$ model and also assume equilibrium chemistry rather than finite-rate chemistry.

Figure 6 plots the temperature profiles along the stagnation streamline for the MUSES-C subscale model, showing the limited effect of including radiative cooling. This reinforces the earlier findings in Section 3.1 where it was shown that at the small radii the radiative heat transfer became quite a small proportion of the total heat load. The implications for experimenters are that it will require very sensitive measurement techniques to detect the radiative heat transfer when it is about $1 \%$ of the total heat transfer to the vehicle.

As noted earlier, these results should be interpreted as preliminary only. The problems of sufficient grid resolution in the boundary layer are not fully addressed. We wish also, in the future, to use a shock-fitting method in preference to the present shock-capturing scheme. This may simultaneously help with getting better resolution in the boundary layer and avoid some of the numerical errors introduced by the directionally-split flux calculators 
Temperature along stagnation streamline

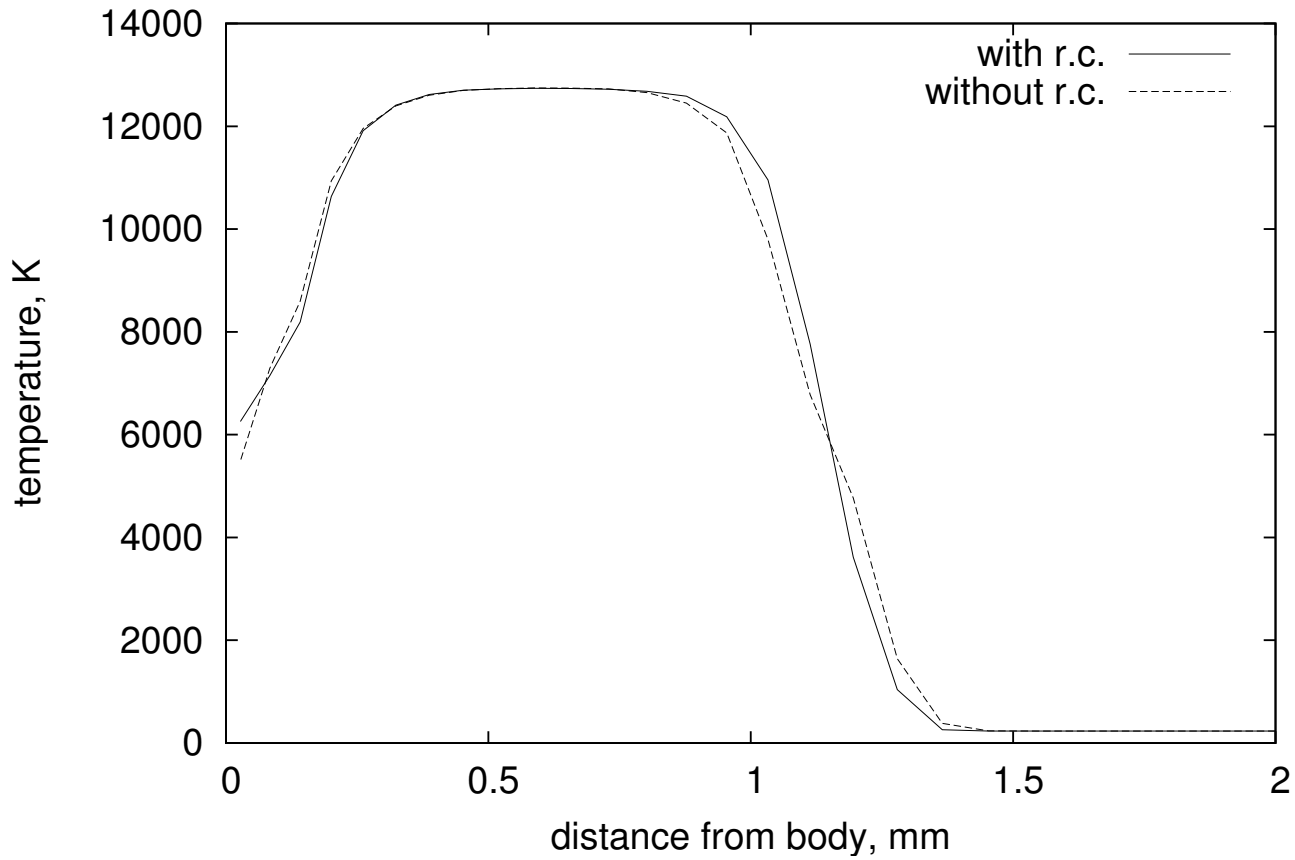

Figure 6: Temperature profiles for the MUSES-C 1:10 scale model: $\mathrm{p}_{\infty}=$ $110.12 \mathrm{~Pa}, \mathrm{~T}_{\infty}=233.25 \mathrm{~K}, \mathrm{~V}_{\infty}=11.6 \mathrm{~km} / \mathrm{s}$ r.c. $=$ radiative cooling 
computing flow through the very strong bow shock.

\section{Concluding Remarks}

The simulations presented here treated hypersonic flows about axisymmetric bodies with viscous and radiative effects included. The main motivation for the work was to identify what radiative cooling effects would occur for subscale models used in the expansion tubes at the University of Queensland. The model for the radiative heat transfer in the flow was a transparent gas which, although simple, was shown to be accurate enough for these preliminary calculations.

The results show that measurements of total heat transfer to the surface of the vehicle and the shock stand-off distance are poor indicators of the presence of radiation in the subscale flowfields at relatively high densities. In terms of heat transfer, it should be possible to measure the total heat transfer and the radiative heat transfer by separate methods [13] and assess the relative contribution of convection and radiation to the heat transfer.

Future work in this area would best focus on a more physically realistic model for the radiative heat transfer in the flow and the method of coupling the radiation effect to gas dynamics. This should allow for more accurate quantitative analysis of the various flow features and could lead to better correlations for hypersonic blunt body flowfields with radiation effects included. This would have application to the design studies for the Titan mission where the $\mathrm{N}_{2}+\mathrm{CH}_{4}$ atmosphere is expected to radiate strongly [11].

On issues of numerical implementation, the method of coupling leads to a problem in that the radiative transfer may appear in the gas dynamics energy equation as a stiff source term. It appears that we are fortunate in the present work with our choice of flow conditions but a more robust method would need to address the stiff source term issue. 


\section{References}

[1] J. D. Anderson, Jr. Nongray radiative transfer effects on the radiating stagnation region shock layer and stagnation point heat transfer. Aerodynamics Research Report No. 281 NOLTR 67-104, U.S. Naval Ordnance Laboratory, 1967. C250, C253, C256, C259

[2] J. D. Anderson, Jr. Hypersonics and High Temperature Gas Dynamics. McGraw Hill, 1989. C252, C259

[3] R. Doihara and M. Nishida. Viscous shock layer studies of a super orbital reentry capsule using a multi vibrational temperature model. Report SP No. 17, The Institute of Space and Astronautical Science, 2003. C261, C263

[4] R. J. Gollan and P. A. Jacobs. Numerical modelling of radiating superorbital flows: with detailed governing equations. Division of Mechanical Engineering Report, The University of Queensland, Brisbane, December 2003. C252

[5] P. A. Jacobs. MB_CNS: A computer program for the simulation of transient compressible flows. Department of Mechanical Engineering Report 10/96, The University of Queensland, Brisbane, December 1996. C252

[6] S. Matsuyama, Y. Shimogonya, N. Ohnishi, and K. Sawada. Numerical simulation of Galileo probe entry flowfield with radiation. AIAA Paper 2002-2994, 2002. C250

[7] T. J. McIntyre, M. J. Wegener, A. I. Bishop, and H. Rubinzstein-Dunlop. Simultaneous two-wavelength holographic interferometry in a superorbital expansion tube facility. Applied Optics, 36(31):8128-8134, 1997. C251 
[8] R. G. Morgan, A. Sasoh, B. Littleton, T.J. McIntyre, J.D. Hoogland, and A.D. Gardner. Simulation of ablative mixing layers in superorbital flows. In 22nd International Symposium on Shock Waves, number 1358, pages 727-732, 1999. C250, C256, C261

[9] A. J. Neely and R. G. Morgan. The Superorbital Expansion Tube concept, experiment and analysis. The Aeronautical Journal, 98(973):97-105, 1994. C251

[10] W. B. Olstad. Stagnation-point solutions for an inviscid radiating shock layer. In Proceedings of the 1965 Heat Transfer and Fluid Mechanics Institute, pages 138-156. Stanford University Press, 1965. $\mathrm{C} 253$

[11] J. Olejniczak, M. Wright, D. Prabhu, N. Takashima, B. Hollis, V. Zoby, and K. Sutton. An analysis of the radiative heating environment for aerocapture at Titan. AIAA Paper 2003-4953, 2003. C250, C265

[12] H. Otsu, K. Suzuki, K. Fujita, and T. Abe. Assessment of forebody and backbody radiative heating rate of hypervelocity reentry capsule. Report SP No. 17, The Institute of Space and Astronautical Science, 2003. C261

[13] R. A. Palmer. Measurement of heat transfer in superorbital flows. PhD thesis, Department of Mechanical Engineering, University of Queensland, 1999. C250, C262, C265

[14] R. J. Stalker. Hypervelocity aerodynamics with chemical nonequilbrium. Annual Review of Fluid Mechanics, 21:37-60, 1989. $\mathrm{C} 261$

[15] K. Sutton. Air radiation revisited. In Thermal Design of Aeroassisted Orbital Transfer Vehicles, volume 96 of Progress in Astronautics and Aeronautics, pages 419-441. AIAA, New York, 1985. C250 
[16] M. E. Tauber and K. Sutton. Stagnation-point radiative heating relations for Earth and Mars entries. A.I.A.A. Journal of Spacecraft and Rockets, 28(1):40-42, 1991. C259, C262

[17] E Zoby. Empirical stagnation-point heat-transfer relation in several gas mixtures at high enthalpy levels. Technical Report TN D-4799, NASA, 1968. C262 